\title{
STARBURSTS TRIGGERED BY CLOUD COMPRESSION IN INTERACTING GALAXIES
}

\author{
CHANDA J. JOG \\ Department of Physics, Indian Institute of Science \\ Bangalore 560012, India
}

\begin{abstract}
We propose a physical mechanism for the triggering of starbursts in interacting spiral galaxies by shock compression of the pre-existing disk giant molecular clouds (GMCs). We show that as a disk GMC tumbles into the central region of a galaxy following a galactic tidal encounter, it undergoes a radiative shock compression by the pre-existing high pressure of the central molecular intercloud medium. The shocked outer shell of a GMC becomes gravitationally unstable, which results in a burst of star formation in the initially stable GMC. In the case of colliding galaxies with physical overlap such as Arp 244, the cloud compression is shown to occur due to the hot, high-pressure remnant gas resulting from the collisions of atomic hydrogen gas clouds from the two galaxies. The resulting values of infrared luminosity agree with observations. The main mode of triggered star formation is via clusters of stars, thus we can naturally explain the formation of young, luminous star clusters observed in starburst galaxies.
\end{abstract}

\section{Starburst Galaxies: Background}

Starburst galaxies are characterized by a high infrared luminosity, $\sim 10^{10}-$ $10^{12} L_{\odot}$, seen typically over the central region of $\sim 1 \mathrm{kpc}$ diameter; and they display a strong central concentration of molecular hydrogen gas. The infrared luminosity is attributed to a high rate of formation of massive stars. The stellar radiation is absorbed by dust in the gas clouds and re-radiated thermally in the infrared.

Starbursts are most often observed in interacting field galaxy pairs and barred galaxies, hence the interaction or the bar must be important in the triggering of the starbursts. For a brief review of the physical properties 
of starburst galaxies, as well as their implications for related fields, see for example Jog (1995).

\subsection{PHYSICAL MECHANISM FOR TRIGGERING OF STARBURSTS}

Ever since the discovery of starbursts, the triggering process for them has remained an important and a challenging problem. To understand the starburst phenomenon, it is useful to compare it with star formation in a normal, quiescent galaxy like the Milky Way galaxy. The molecular hydrogen gas in the galactic disk is distributed in giant molecular clouds (GMCs) with masses $\sim 10^{5}-10^{6} M_{\odot}$ each. A typical GMC is self-gravitating and is in a near-virial equilibrium, and forms the site of massive-star formation but has a low star formation rate. Thus, the starburst galaxies pose two puzzles. First, what is the triggering mechanism for the observed burst of efficient star formation? We have addressed this question. Second, what is the origin of the central concentration of gas? Work on this question by a number of authors over the last ten years has clearly established that a galactic tidal interaction or a bar increases the dissipation in gas and this causes most of the gas from a galactic disk to fall into the central regions of the galaxy on timescales greater than the dynamical timescale (e.g., Norman 1992). The infall timescales are $\sim 3 \times 10^{8} \mathrm{yr}$ for tidally interacting galaxies and $\sim 10^{7} \mathrm{yr}$ for evolved mergers, and we use these values in our model. However, these gas infall models do not consider the physical details of triggering of star formation. Some of these models assume that cloud collisions enhance the star formation rate but do not give the details of triggering.

We have addressed the above first question of triggering of starbursts. We propose a novel yet simple physical mechanism for the triggering of starbursts by shock compression via high external pressure on a pre-existing disk GMC. The basic idea behind our mechanism is as follows. In an isolated galaxy, a disk molecular cloud is barely stable to begin with. If the ambient pressure around the GMC increases, this overpressure can cause a radiative shock compression of the outer layers of the GMC. The shocked outer shell of a GMC is shown to become gravitationally unstable, resulting in a burst of star formation. We treat the following two cases with different sources of high ambient pressure, these cover the different observed locations of starbursts in starburst galaxies:

Case 1: In tidally interacting galaxies and evolved mergers, the starbursts are observed to occur in the central regions of galaxies. Here the cloud compression is shown to occur due to the pre-existing, high-pressure central intercloud medium (Jog \& Das 1992; Jog \& Das 1996) - see Section 2.

Case 2: In the early stages of a physical collision between galaxies, the 
starbursts are observed to lie in the overlapping wedge regions. Here the cloud compression is shown to occur due to the hot, high-pressure remnant gas resulting from the collisions of atomic hydrogen gas clouds from the two galaxies (Jog \& Solomon 1992) - see Section 3.

This is a simple physical mechanism, and inevitable, given the preexisting high-pressure reservoir of the central intercloud medium in case 1 , and the generation of the hot, high-pressure gas in case 2 . In both the cases, we consider a galaxy with pre-encounter interstellar medium parameters as observed in the Milky Way galaxy, and then study the detailed evolution of this realistic interstellar medium following a galaxy encounter.

\section{Triggering of Starbursts by Central Overpressure}

The physical outline of the triggering mechanism for central starbursts in interacting galaxies (Jog \& Das 1992) is as follows. The observations of the central disk region of $\sim 1 \mathrm{kpc}$ diameter of the Galaxy (Bally et al. 1988) have shown the existence of a molecular intercloud medium (ICM), with a high average molecular hydrogen number density of $50 \mathrm{~cm}^{-3}$. The ICM is distributed uniformly with a large volume filling factor $\sim 1$; and also has high internal, non-thermal random motions. The physical properties of the central molecular gas are very different from the molecular gas in the galactic disk (also see Section 2.2). We note that the average pressure in the central ICM in the Galaxy, $P_{\mathrm{ICM}}$, is about $\sim 10$ times higher than $P_{\mathrm{GMC}}$, the effective non-thermal gas pressure inside a disk GMC.

We show that as a typical disk GMC tumbles into the central region of the galaxy following a galactic tidal encounter or due to a bar, the pre-existing high pressure of the central ICM drives a strong isothermal/radiative spherical shock into the cloud. Hence, the post-shock gas accumulates into a thin, dense shell which moves inwards with a velocity equal to the shock velocity. The initial shock velocity is set by the ambient pressure of the central ICM, and at later stages the self-gravity of the shell accelerates the shell inwards. On solving the equation of motion of the shocked shell, we obtain $t_{\mathrm{c}}(r)$, the time taken for a shock to cross to a radius $r\left(<R_{\mathrm{c}}\right)$ from $R_{\mathrm{c}}$, where $R_{\mathrm{c}}$ is the radius of the cloud. The crossing time, $t_{\mathrm{c}}$, increases as the shock is driven into the cloud.

The shocked shell of a GMC can develop gravitational instabilities. The stability of the thin, dense shell is analyzed using a plane-parallel geometry for the shell and a local, linear perturbation analysis - exactly as in the case of pressure-supported thin disks. This gives $t_{\mathrm{g}}$, the growth time for the fastest growing mode, to be inversely proportional to the surface density of the shocked shell. Hence as the shock moves in and more mass is swept into the shell, $t_{\mathrm{g}}$ decreases. 


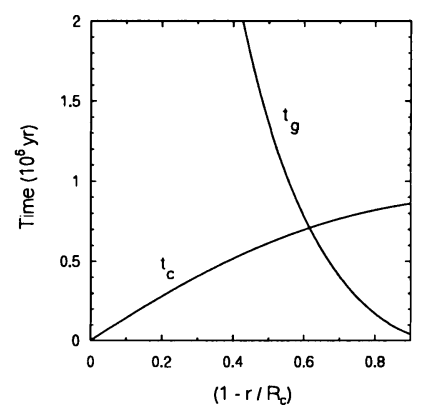

Figure 1. A plot of $t_{\mathrm{c}}$, the shock crossing time, and $t_{\mathrm{g}}$, the growth time for the fastest growing gravitational instability in the shocked shell, vs. the fractional cloud penetration depth. The instabilities set in when $t_{\mathrm{g}}=t_{\mathrm{c}}$; here $f_{\text {shell }}=0.85$ (from Jog \& Das 1996).

The cloud compression continues until $t_{\mathrm{g}}$ becomes smaller $t_{\mathrm{c}}$; at this point the shocked shell becomes gravitationally unstable and begins to fragment (Fig. 1). Further non-linear evolution of this fragmenting, highdensity shell results in star formation. We define $f_{\text {shell }}$ to be the fraction of the cloud mass that is compressed into a shocked shell at the onset of gravitational instabilities. We obtain $f_{\text {shell }}=0.85$ for the typical disk GMC and the central ICM parameters in the Galaxy.

The star formation in the shocked, high-density gas in the GMC shell is characterized by a high star formation or conversion efficiency, and a preferential formation of massive stars of a few $M_{\odot}$ each (Larson 1987; Jog \& Solomon 1992). The net central infrared luminosity is shown to be linearly proportional to the fraction of cloud mass compressed $\left(f_{\text {shell }}\right)$, the efficiency of star formation in the shocked gas, and the rate of gas infall from the disk. The last factor depends only on the strength of the encounter and on the initial radial distribution of gas in the galaxy, whereas $f_{\text {shell }}$ depends on the microscopic physics of cloud compression. It is interesting that the two effects are decoupled.

For a tidal/distant encounter or a barred galaxy, the resulting lower limits on the central infrared luminosity and the ratio of the infrared luminosity-to-gas mass are $\sim 6 \times 10^{9} L_{\odot}$ and $\sim$ a few $L_{\odot} / M_{\odot}$ respectively. These results agree reasonably well with the observations of starburst galaxies such as M82 or NGC 253. The evolved mergers of galaxies, with their higher central gas concentrations, yield higher values of $\leq 10^{12} L_{\odot}$ and $\leq 100 L_{\odot} / M_{\odot}$, respectively. These agree with the observed values from ultraluminous galaxies such as Arp 220 or NGC 6240.

Our study has shown the viability of such a triggering mechanism. Future work should take account of the observed internal clumpy structure of clouds, and consider the shock formation in a clumpy cloud. 


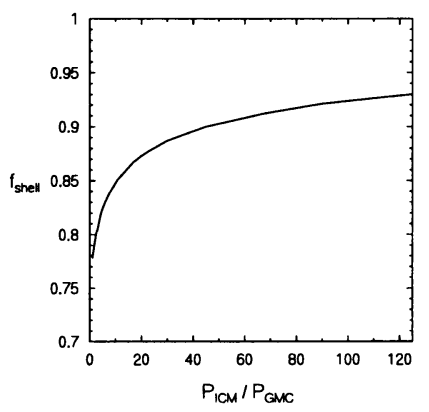

Figure 2. A plot of $f_{\text {shell, }}$, the fraction of cloud mass compressed at the onset of gravitational instabilities in the shell, vs. the ICM overpressure $P_{\mathrm{ICM}} / P_{\mathrm{GMC}}$ (from Jog \& Das 1996).

\subsection{A PARAMETER STUDY FOR CENTRAL STARBURSTS}

Recent observations have shown the existence of a central molecular intercloud medium (ICM) with high pressure also in IC 342 (Downes et al. 1992), NGC 1808 (Aalto et al. 1994), Arp 220, and Mrk 231 (Downes \& Solomon 1997). Thus the high-pressure central ICM seems to be a common gas component of spiral galaxies and especially of starburst galaxies.

Therefore, we have now done a parameter study of cloud compression which covers a wide range of realistic values for the disk cloud and the central ICM gas parameters. These cover a range for the ICM overpressure, $P_{\mathrm{ICM}} / P_{\mathrm{GMC}}$, from a few to 125 . The aim was to see under which conditions shock compression of a GMC can occur (Jog \& Das 1996). We show that $f_{\text {shell }}$, the fraction of cloud mass shocked at the onset of gravitational instabilities, depends only on the external ICM pressure, the cloud density, and the cloud radius. We find that $f_{\text {shell }}$ is high $\sim 0.75-0.90$, and is not sensitively dependent on the value of the ICM overpressure (Fig. 2). This somewhat surprising result comes about because the initial inward shock velocity is only proportional to the square root of the ICM pressure, hence even a small overpressure is sufficient to start the process of compression. At later stages of compression, the shock velocity is mainly set by the self-gravity of the shell, hence this process eventually results in the compression of a substantial fraction of the cloud mass. This is an important result because it shows that the starbursts triggered by central overpressure as proposed by Jog \& Das (1992) is a general triggering mechanism.

Applying these results to IC 342 and NGC 1808, we show that in both cases $f_{\text {shell }}>0.9$. However, the net central luminosity from star formation depends on the gas infall rate. Thus, NGC 1808 with gas infall due to tidal interaction with NGC 1792 shows a strong central starburst, while IC 342 
with a lower gas infall rate due to a weak bar shows only a moderate central starburst. Thus, all large, gas-rich spiral galaxies appear to be poised on the brink of starburst, and whether or not a strong central starburst occurs depends on the rate of gas infall from the galactic disk.

\subsection{CENTRAL MOLECULAR INTERCLOUD MEDIUM - ICM}

Our mechanism for triggering of starbursts via central overpressure (Jog \& Das 1992; Jog \& Das 1996) has highlighted the diffuse molecular ICM in the central regions of galaxies. The high-pressure ICM appears to be a common feature of spiral galaxies, and contains a substantial fraction of the central gas mass. Therefore it is important to understand its origin, energetics, and also its evolution following a starburst, as was stressed by Jog \& Das (1992). The ICM is not self-gravitating but is instead probably confined by the galactic gravitational potential. The origin and the physical properties of the ICM seem to be determined by its unique central location in a galaxy. For example, the ICM can originate due to the strong central galactic tidal fields, especially in a barred potential (Das \& Jog 1995); and also due to the high central, non-thermal random motions, which can result in disruptive cloud collisions.

To formulate these complex problems theoretically, we need to have more high-resolution, multi-transition m.m. wave data for different types of galaxies, and for starburst galaxies in different stages of evolution - as in Aalto et al (1994); and Downes \& Solomon (1997).

\section{Triggering Of Starbursts In Colliding Galaxies}

The second case of triggering of starbursts involves the early stages of a physical collision/merger between two field spiral galaxies (Jog \& Solomon 1992). We consider the detailed evolution of a realistic interstellar medium consisting of $\mathrm{H}_{2}$ and $\mathrm{HI}$ in a galaxy during a galaxy collision. The $\mathrm{HI}$ clouds from the two galaxies collide; whereas the GMCs, with a mean free path typically larger than the size of the overlapping region, do not collide. Since the H I clouds collide at the typical relative velocity between field galaxies $\sim 300 \mathrm{~km} \mathrm{~s}^{-1}$, their kinetic energy of relative motion is thermalized and this results in a hot, ionized, high-pressure remnant gas. This gas has a pressure $\sim 200$ times higher than $P_{\mathrm{GMC}}$, the effective internal pressure in a disk GMC, hence it causes a radiative shock compression of the pre-existing GMCs in the overlapping wedge region. This results in a burst of massivestar formation in the shocked outer shells of GMCs, with the details of star formation the same as in Jog \& Das (1992). In this case the hot remnant gas cools fast and hence the overpressure lasts for a short time, whereas in 
the earlier case the central ICM constitutes an "everpresent" high-pressure reservoir. The main results from this study are:

1. The starbursts occur in situ in regions of overlap/interpenetration between the two colliding galaxies. Thus we can naturally explain the starbursts observed in the regions of disk overlap as in Arp 244 (NGC 4038/39), and in Arp 299 (NGC 3690/IC 694).

2. The duration of starburst is equal to the disk crossing time $\sim$ a few $\times 10^{7} \mathrm{yr}$. Hence in a region of overlap, we would expect to see the progression of starburst on this time scale. It is interesting that exactly such age gradients have now been observed in Arp 244 (Vigroux 1997), and also for the high redshift galaxies (Abraham 1997).

3 . Whether the central starburst or the extranuclear disk case is stronger depends on the geometry and the epoch of collision and also on the initial radial distribution of gas. In general, because of the higher geometric crosssection for a disk overlap, a starburst would occur there at first (as is observed in Arp 244), to be followed later on by a stronger central starburst.

4. We predict the formation of young globular clusters via shock compression of pre-existing GMCs in colliding galaxies (also see Section 4).

5. The absence of super-starbursts in the cluster galaxies at the present epoch is explained due to the high speed collisions that occur between cluster galaxies (Valluri \& Jog 1990).

\section{Formation of Young, Luminous Star Clusters}

An important feature of our triggering mechanism is that the triggered star formation occurs via gravitational instabilities in the shocked outer shells of GMCs. Thus, the main mode of star formation is via formation of clusters of stars, which are gravitationally bound to begin with. Hence our model provides a physical mechanism for the formation of young globular or populous star clusters as we had proposed in Jog \& Solomon (1992).

The study of the detailed shock propagation in a cloud and the complete coverage of the parameter space has now allowed us to obtain the detailed physical properties of such star clusters (Jog \& Das 1996). The mass of an instability, $M_{\text {inst }}$, formed in the shocked outer shell of a GMC gives an upper limit on the mass of the star cluster formed. The star clusters formed are predicted to be flattened. We have obtained $M_{\text {inst }}$ as a function of the disk cloud mass and the central ICM pressure. We find that $M_{\text {inst }}$ is a large fraction $(\sim 0.3-0.5)$ of the GMC mass. This is because the fraction of a cloud mass shocked $\left(f_{\text {shell }}\right)$ is always high, and the number of fragments or instabilities per cloud is small. Thus, in our model of triggering by cloud compression, an efficient star formation is triggered simultaneously in a 
sizeable fraction of the cloud mass. The upper limit on the mass of the star clusters formed covers a range of values $\sim 10^{4}-10^{6} M_{\odot}$.

There is a growing observational evidence especially from the $H S T$, for the existence of young, luminous star clusters or super star clusters in starburst galaxies - as for example, in Arp 244 (Whitmore \& Schweizer 1995), and in NGC 3921 (Schweizer et al. 1996). Our triggering mechanism provides a natural explanation for the origin of these young star clusters. Recently Ho \& Filippenko (1996) have made direct, dynamical mass measurements for two young star clusters, one each in the dwarf galaxies NGC 1569 and NGC 1705 , to be equal to $3.3 \times 10^{5} M_{\odot}$ and $0.8 \times 10^{5} M_{\odot}$, respectively. These lie in the range of masses of star clusters resulting from our mechanism. Also, the observed sizes of these $(\sim$ a few pc) agree with the sizes of instabilities obtained from our model. Thus, we can now attempt to compare the quantitative predictions from our model with the new high-resolution data on the young star clusters.

Acknowledgements: It is a pleasure to acknowledge the collaboration, and also more recent discussions, with Mousumi Das and Phil Solomon. I would like to thank the IAU, the Indian Institute of Science, and the Indian National Science Academy for financial support which enabled me to attend this exciting meeting.

\section{References}

Aalto, S., Booth, R.S., Black, J.H., Koribalski, B., \& Wielebinski, R. 1994, $A$ \& $A$, 286, 365

Abraham, R.G. 1997, these proceedings

Bally, J., Stark, A.A., Wilson, R.W., \& Henkel, C. 1988, ApJ, 324, 223

Das, M. \& Jog, C.J. 1995, ApJ, 451, 167

Downes, D., Radford, S.J.E., Guilloteau, S., Guelin, M., Greve, A., \& Morris, D. 1992, $A \& \mathcal{E} A, \mathbf{2 6 2}, 424$

Downes, D., \& Solomon, P.M. 1997, to appear in ApJ

Ho, L.C., \& Filippenko, A.V. 1996, ApJ, 472, 600

Jog, C.J. 1995, BASI, 23, 135

Jog, C.J., \& Das, M. 1992, ApJ, 400, 476

Jog, C.J., \& Das, M. 1996, $A p J$, 473, 797

Jog C.J., \& Solomon, P.M. 1992, ApJ, 387, 152

Larson, R.B. 1987, in Starbursts and Galaxy Evolution, eds. T.X. Thuan, T. Montmerle, \& J. Trann Thanh Van, Edition Frontieres, Paris, 467

Norman, C.A. 1992, in Massive Stars in Starbursts, eds. C. Leitherer, T.M. Heckman, C.A. Norman, \& N.R. Walborn, Cambridge Univ. Press, Cambridge, 271

Schweizer, F., Miller, B.W., Whitmore, B.C., \& Fall, S.M. 1996, AJ, 112, 1839

Valluri, M., \& Jog, C.J. 1990, ApJ, 357, 367

Vigroux, L. 1997, these proceedings.

Whitmore, B.C., \& Schweizer, F. 1995, AJ, 109, 960 